\title{
Use of a Mobile Telemedicine System during the Transport of Emergency Myocardial Infarction Patients Would Be an Effective Technology in the Pre-hospital Medical Setting
}

\author{
Hiroyuki Yokoyama, ${ }^{1,2}$ Nobuhito Yagi, ${ }^{2}$ Yoritaka Otsuka, ${ }^{2}$ Jun-ichi Kotani, ${ }^{2}$ Masaharu Ishihara, ${ }^{2}$ \\ Satoshi Yasuda, ${ }^{2}$ Kazuhiro Sase, ${ }^{3}$ Hisao Ogawa, ${ }^{2}$ Hiroshi Nonogi ${ }^{2}$
}

\begin{abstract}
Background: We have developed a mobile telemedicine system (MTS) utilizing mobile communications via cellular phones, making it possible to continuously transmit biological information, including 12-lead electrocardiography data, from an ambulance while the patient is being transported to the destination hospital in real time. Purpose: We evaluated whether using an MTS during the transport of acute myocardial infarction patients shortens the interval between arrival at the hospital and balloon inflation to achieve reperfusion (doorto-balloon time). Two hundred eighteen consecutive AMI patients were divided into two groups: 23 patients who had been brought to the hospital in an ambulance equipped with an MTS, and 195 patients who had been brought to the hospital without the use of an MTS. Results: When the MTS group and the non-MTS group were compared, no differences in clinical characteristics, Killip class, incidence of emergency coronary angiography, culprit lesion, or prevalence of multi-vessel disease were seen. No significant differences were seen between the two groups in terms of the incidence of primary percutaneous coronary intervention (PCl), initial angiography findings, or the degree of coronary blood flow after PCl. Regarding outcomes, no differences in the peak creatinine kinase and isozyme MB levels or in-hospital mortality were seen between the two groups. However, the door-to-balloon time was 86 minutes (median times) in the MTS group, which was significantly shorter than the 96 minutes observed in the non-MTS group $(P<0.05)$. Conclusions: We have developed a mobile telemedicine system (MTS) and have shown its efficacy in pre-hospital medical settings. KEY WORDS: mobile telemedicine system, door-to-balloon time, pre-hospital care
\end{abstract}

\section{Background}

It is not rare for patients with acute myocardial infarction (AMI) to die suddenly outside the hospital or for their condition to become more serious as a result of delays in treatment, and setting up an effective pre-hospital medical system before admission to a hospital is an urgent task for reducing the AMI-related mortality rate. Because half of all patients who die as a result of AMI do so before reaching a hospital, the 2010 International Consensus on Cardiopulmonary Resuscitation and Emergency Cardiovascular Care Science (G2010) advised that the interval between emergency medical service (EMS) contact with a patient and balloon inflation to achieve reperfusion (EMS-toballoon time) should be no more than 90 minutes. Also, the

${ }^{1}$ Kadowaki Clinic, 132-6 Kaminomoto, Higashimatsuyama, Saitama 355-0073, Japan, ${ }^{2}$ Division of Cardiovascular Care Unit, National Cerebral and Cardiovascular Center, ${ }^{3}$ Department of Clinical Pharmacology, Juntendo University

Received July 2, 2013; Accepted December 26, 2013

doi: 10.7793/jcoron. 20.13-00011
American College of Cardiology (ACC)/American Heart Association (AHA) Task Force on Practice Guidelines ${ }^{1)}$ recommends minimizing the total ischemic time and a first medical contactto-balloon time of within 90 minutes for patients with AMI. Although pre-hospital 12-lead electrocardiogram (ECG) recordings can provide advance information to the hospital, it is important to shorten both the time between the arrival of the patient at the hospital and the recording of the first ECG as well as the time between the ECG recording and the start of reperfusion therapy, ${ }^{2-5)}$ a practice that has not been widely adopted in Japan. We have developed a mobile telemedicine system (MTS) ${ }^{6}$ that utilizes mobile communications via cellular phones, making it possible to transmit biological information, including 12-lead ECG data, blood pressures, respiration rates, pulse rates and in-vehicle images acquired with a small camera, continuously from an ambulance while the patient is being transported to the destination hospital in real time.

We have started to apply our newly developed MTS, which continuously transmits biological information from ambulances in real time, and tested the validity of this technology in a pre- 
hospital medical setting, in addition to using the MTS clinically while transporting patients with AMI to investigate its effectiveness for shortening the interval between arrival at the hospital and balloon inflation to achieve reperfusion (door-to-balloon time).

\section{Methods}

\section{Mobile telemedicine system}

The effects of vibrations during ambulance transport and the person's body movements were eliminated from the 12-lead ECG using the motion noise reduction function of the Radarcirc ${ }^{\circledR}$ electrocardiograph (Dainippon Sumitomo Pharma Co., Ltd) in our MTS. The NTT Comware Corporation's L-Box ${ }^{\circledR}$ ultra-small Linux server was used for the data transmission, and biological information was sent via the Internet through a FOMA (Freedom of Mobile Multimedia Access) line and a PDA (personal digital assistant) for information control. We used the standard specification MFER (Medical waveform Format Encoding Rules) ${ }^{7)}$ for the medical waveform data used for ECG data transmission, which enabled biological information for medical use to be transmitted as compressed information that had been standardized and encrypted. We used third-generation cellular telephones to perform a bench test to confirm the accuracy of the real-time continuous transmission of the 12-lead ECG monitoring and images via the Internet. Then, we used the MTS to conduct a biological information transmission experiment from a moving ambulance and confirmed that it took 45 seconds to start up the transmission system in the ambulance, 45 seconds (20-70 seconds) after pushing the 12-lead "send" button for the 12-lead ECG to begin to be displayed at the hospital, and 3 seconds (1-5 seconds) after starting to send the monitor images for the images to be displayed on the hospital screen. The field trial confirmed that there were no problems with transmitting biological information, including continuous 12-lead ECG monitor waves, blood pressures, respiration rates, pulse rates or in-vehicle images, from the ambulance to the hospital in real time while driving on ordinary roads or expressways, nor were there any problems with the data or images transmitted from either ordinary roads or expressways, with remote camera operation, or with line reconnection functions. The transmission delay time was confirmed to be 10 to 12 seconds for the 12-lead ECG monitor waves, and the camera images had a resolution of $320 \times 240$ pixels and were transmitted at approximately 2-3 frames/second. The MTS was installed in six fire department ambulances in Suita city and was used at the discretion of EMS members when a cardiovascular emergency patient was expected.

\section{Study populations}

Two hundred eighteen consecutive AMI patients, including patients who requested EMS assistance 24 hours after the onset of AMI, who were brought to our hospital by ambulance between June 2008 and October 2009 were included in the analysis. The subjects were divided into two groups according to whether they had or had not been brought to the hospital in an ambulance equipped with an MTS (MTS group, $n=23$; non-MTS group, $n=195$ ). The door-to-balloon time was then investigated and compared.

\section{Coronary angiography}

"Significant narrowing" was defined as a narrowing of the vessel by $75 \%$ or more, and "multi-vessel disease" was defined as a lesion in two or more major branches. "Emergency coronary angiography" was defined as coronary angiography performed by directly proceeding to the coronary angiography room from the emergency room. The coronary artery responsible for the AMI was identified based on coronary angiography, ECG, and echocardiography findings. The performance of percutaneous coronary intervention (PCI) was left to the discretion of the operator.

\section{Statistical analysis}

All the statistical analyses were performed using statistical software program packages (JMP software version 8.0J and SAS 9.1, SAS Institute, Cary, NC).

\section{Ethical considerations}

This study was conducted in accordance with the ethical guidelines for epidemiological studies and was approved by the ethics committee of the National Cerebral and Cardiovascular Center.

\section{Results}

In the field trial, we confirmed the application of motion noisereduction, in which adaptive filters are used for every beat and each filtered ECG is connected smoothly using a weighted mean method (Fig. 1). In the final ECG, the ECG wave line was distorted, rather than the other ECG wave. In Fig. 1, column 1 shows the original ECG wave from a volunteer who intentionally twisted his/her upper body while being transported in the moving vehicle, and column 2 shows the filtered ECG wave with the adapted filtering and weighted mean method.

Fig. 2 shows the ECG changes obtained by MTS during transportation in a very representative clinical case. This patient had complained of chest pain and requested EMS assistance; the first ECG data was sent to the hospital at 12:08 am from the moving ambulance and showed a mild ST depression in the limb leads (Fig. 2A). However, 7 minutes later, while the patient was still be transported to hospital, the patient developed a more severe chest pain and the ECG data showed a worsening of the ST depression in the precordial leads (Fig. 2B). After admission, this patient underwent coronary angiography, which revealed severe disease involving 3 vessels; the patient was successfully treated 


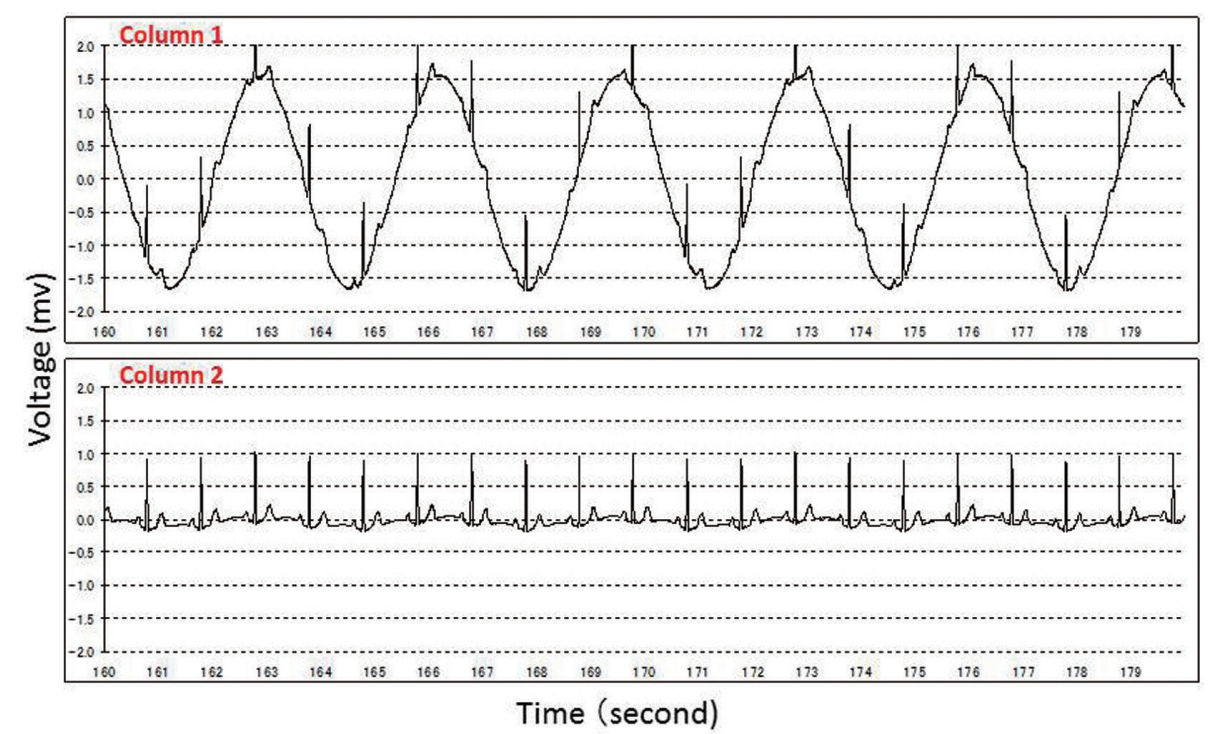

Fig. 1 Motion noise-reduction system.

Column 1 shows the original ECG wave from a volunteer who intentionally twisted his/her upper body while being transported in the moving vehicle, and column 2 shows the filtered ECG wave with the adapted filtering and weighted mean method.

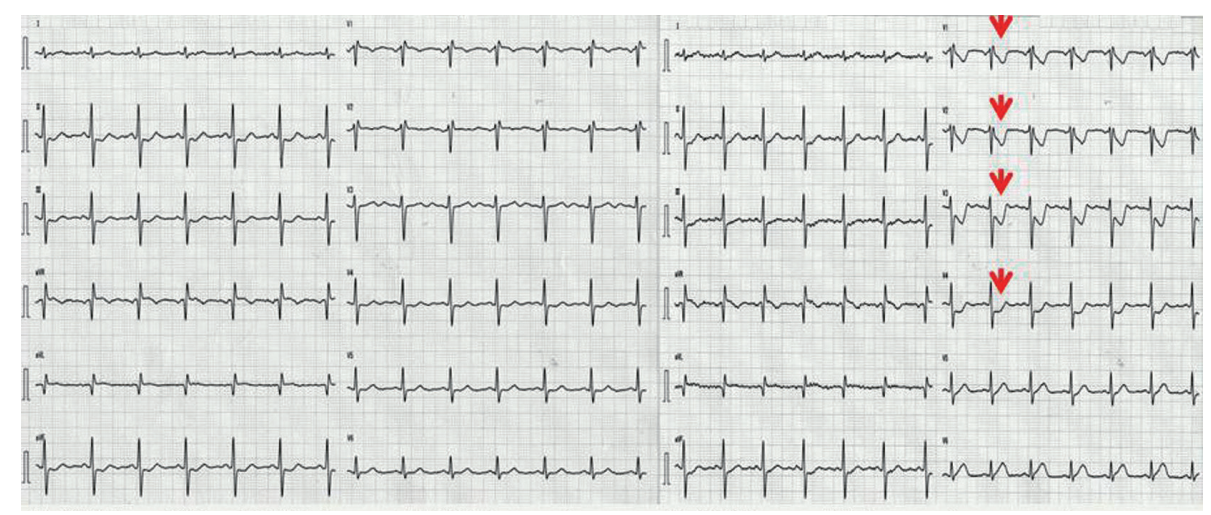

Fig. 2 The ECG obtained by MTS during transportation.

The first ECG data was sent to the hospital from the moving ambulance and showed a $A \mid B$ mild ST depression in the limb leads at 12:08 am (Fig. 2-A), the ECG data showed a worsening of the ST depression in the precordial leads after 7 minutes at 12:15 am (Fig. 2-B).

using PCI.

During this study period, 218 consecutive AMI patients were brought to our hospital by ambulance and were divided into two groups according to whether they had been brought to the hospital in an ambulance equipped with or without an MTS (MTS group, $n=23$; non-MTS group, $n=195$ ). Twelve-lead ECG data was clearly received from the moving ambulance for all the patients in the MTS group, and Fig. 3 shows a view of the monitor screen for a patient with LMT disease whose biological data was sent by MTS during after-hours. When the MTS group and the non-MTS group were compared, no differences in age, sex, coronary risk factors, or left ventricular ejection fraction were seen (Table 1). In the MTS group, more than $90 \%$ of the patients ex- hibited ST elevation myocardial infarction (STEMI), about half of them had an onset-to-door time of less than 3 hours, and $61 \%$ of them arrived at the hospital during after-hours (18 p.m.-8 a.m.). In the MTS group, 7 patients with STEMI arrived at the hospital less than 3 hours after the onset of AMI during the hospital's after-hours.

Also, no differences in the performance of emergency CAG, the culprit lesion, or the prevalence of multi-vessel disease were seen, and all of the patients in the MTS group underwent primary PCI. No significant differences were observed between the two groups in terms of the performance of primary PCI, the initial angiography findings, or the degree of coronary blood flow after PCI (Table 2). When the outcomes of the two groups were 


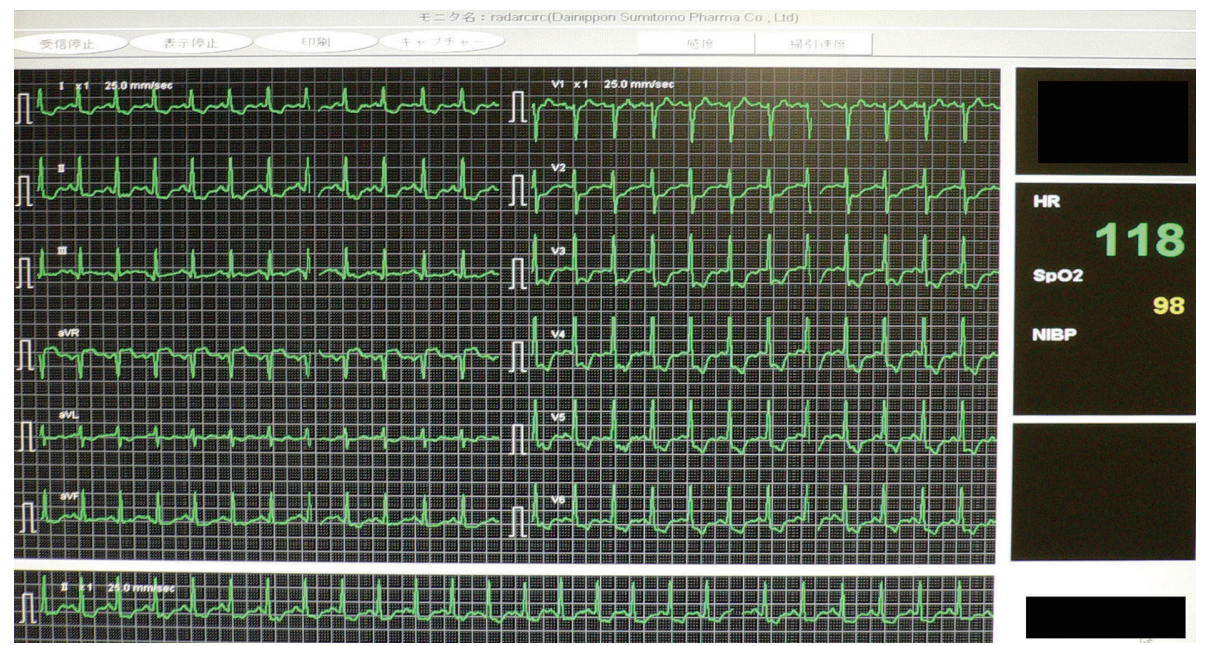

Fig. 3 A view of the monitor screen for a patient.

Table 1 Clinical characteristics

\begin{tabular}{lccc}
\hline & MTS $(\mathrm{n}=23)$ & Non-MTS $(\mathrm{n}=195)$ & $p$ value \\
\hline Age (years) & $67 \pm 9$ & $68 \pm 12$ & $\mathrm{~ns}$ \\
Male, $\mathrm{n}(\%)$ & $18(78)$ & $144(74)$ & $\mathrm{ns}$ \\
Diabetes mellitus, $\mathrm{n}(\%)$ & $11(48)$ & $94(48)$ & $\mathrm{ns}$ \\
Dyslipidemia, $\mathrm{n}(\%)$ & $17(74)$ & $116(59)$ & $\mathrm{ns}$ \\
Hypertension, $\mathrm{n}(\%)$ & $14(61)$ & $130(67)$ & $\mathrm{ns}$ \\
Obesity, $\mathrm{n}(\%)$ & $8(35)$ & $50(26)$ & $\mathrm{ns}$ \\
Smoking, $\mathrm{n}(\%)$ & $6(26)$ & $69(35)$ & $\mathrm{ns}$ \\
Previous MI, $\mathrm{n}(\%)$ & $3(13)$ & $28(14)$ & $\mathrm{ns}$ \\
Killip class, $\mathrm{n}(\%)$ & & & $\mathrm{ns}$ \\
Killip class 1 & $21(9)$ & $183(22)$ & \\
Killip class 2 & $0(0)$ & $7(22)$ & \\
Killip class 3 & $1(9)$ & $17(22)$ & $\mathrm{ns}$ \\
Killip class 4 & $1(9)$ & $172(88)$ & $\mathrm{ns}$ \\
STEMI, n (\%) & $21(91)$ & $108(55)$ & $\mathrm{ns}$ \\
Onset to door time $\leq 3$ hour, n (\%) & $12(52)$ & $96(49)$ & $\mathrm{ns}$ \\
After-hours (18p.m.-8a.m.), n (\%) & $14(61)$ & $44 \pm 11$ & \\
LVEF (\%) & $45 \pm 8$ & & \\
\hline
\end{tabular}

compared, no differences in the peak creatinine kinase levels, creatinine kinase isozyme MB levels, or in-hospital mortality rates were observed (Table 2).

Table 3 shows the door-to-balloon time (median times). Overall, the door-to-balloon time was 86 minutes in the MTS group, which was significantly shorter than the 96 minutes that was observed in the non-MTS group $(\mathrm{P}<0.05)$. Especially for patients with STEMI who arrived at the hospital less than 3 hours after the onset of AMI, the MTS group had a significantly shorter door-to-balloon time than the non-MTS group. Furthermore, the door-to-balloon time tended to be shorter in the MTS group than in the non-MTS group among cases arriving at the hospital dur- ing after-hours (18 p.m.-8 a.m.).

\section{Discussion}

We have developed a mobile telemedicine system (MTS) that continuously transmits biological information from ambulances in real time; here, we have shown the validity of this technology in a pre-hospital medical setting and have confirmed that the use of an MTS while transporting patients with AMI to the hospital enabled the door-to-balloon time to be shortened in a small case series.

In the field trial, we confirmed that the use of an MTS during the transport of patients was an effective technology in a pre- 
J Jpn Coron Assoc 2014; 20: 307-313

Table 2 Coronary characteristic and outcomes

\begin{tabular}{|c|c|c|c|}
\hline & MTS (n=23) & Non-MTS (n=195) & $p$ value \\
\hline Emergency CAG, n (\%) & $23(100)$ & $184(94)$ & ns \\
\hline \multicolumn{4}{|l|}{ Culprit lesions, n (\%) } \\
\hline LAD & $11(48)$ & $77(39)$ & ns \\
\hline LCX & $5(22)$ & $33(17)$ & ns \\
\hline $\mathrm{RCA}$ & $7(30)$ & $64(33)$ & ns \\
\hline LMT & 0 & $13(7)$ & ns \\
\hline Other & 0 & $7(4)$ & $\mathrm{ns}$ \\
\hline \multicolumn{4}{|c|}{ No. of diseased vessels, n (\%) } \\
\hline 1 & $10(43)$ & $67(34)$ & ns \\
\hline 2 & $7(30)$ & $62(32)$ & \\
\hline 3 & $6(26)$ & $60(31)$ & \\
\hline Primary PCI, n (\%) & $23(100)$ & $174(89)$ & ns \\
\hline \multicolumn{4}{|l|}{ Pre TIMI grade, n (\%) } \\
\hline 0 & $13(57)$ & $102(59)$ & ns \\
\hline 1 & $2(9)$ & $8(5)$ & \\
\hline 2 & $4(17)$ & $44(25)$ & \\
\hline 3 & $4(17)$ & $20(11)$ & \\
\hline \multicolumn{4}{|l|}{ Post TIMI grade, n (\%) } \\
\hline 0 & 0 & 2 & ns \\
\hline 1 & 0 & 2 & \\
\hline 2 & $2(9)$ & $8(5)$ & \\
\hline 3 & $21(91)$ & $160(92)$ & \\
\hline CPK (IU/l) & $1525(846,3645)$ & $2243(1131,3866)$ & $\mathrm{ns}$ \\
\hline CK-MB (IU/l) & $202(88,368)$ & $241(119,431)$ & ns \\
\hline Death, n (\%) & $1(4)$ & $14(7)$ & $\mathrm{ns}$ \\
\hline
\end{tabular}

Table 3 Door-to-balloon time

\begin{tabular}{lccc}
\hline & MTS & Non-MTS & $p$ value \\
\hline All cases & $86(63,104)$ & $96(76,130)$ & 0.032 \\
STEMI & $81(60,102)$ & $94(73,125)$ & $\mathrm{ns}$ \\
NSTEMI & $113(104,121)$ & $126(110,196)$ & $\mathrm{ns}$ \\
STEMI and onset to door time $\leq 3$ hours & $69(51,82)$ & $91(70,132)$ & 0.007 \\
STEMI and onset to door time $>3$ hours & $92(80,103)$ & $96(77,132)$ & $\mathrm{ns}$ \\
Working-hours $(8$ a.m.-18 p.m.) & $87(50,114)$ & $95(75,129)$ & $\mathrm{ns}$ \\
After-hours $(18$ p.m.-8 a.m.) & $83(69,103)$ & $97(77,133)$ & 0.07 \\
\hline
\end{tabular}

median (quantile)

hospital medical setting. The motion noise-reduction system in this MTS enabled the ECG data to be transferred smoothly from the moving vehicle to the destination hospital.

In medical literature, shortening the interval between the onset of symptoms and hospital admission has contributed to improving the outcome of patients with AMI. ${ }^{2,8-10)}$ Therefore, recording 12-lead ECG data and providing this information to the destination hospital contributes to shortening the time until the start of reperfusion therapy. ${ }^{11)}$ In Japan, a system in which a pre-hospital 12-lead ECG can be sent by fax has been in use until now, but because the information transmission is limited and the usability of the information at the destination hospitals is also limited, this system has never been widely used. In the STAT-MI study, ${ }^{12,13)}$ it was reported that the time to contact a cardiovascular specialist after the arrival of the EMS team and the time between patient arrival at the hospital and reperfusion were shortened as a result of sending pre-hospital 12-lead ECG data in the form of a PDF file in advance. Moreover, a study in Denmark reported that the door-to-balloon time was shortened by telephotographing a prehospital 12-lead ECG in advance of hospital arrival. ${ }^{14)}$ However, according to that report, VF/VT was observed in $6 \%$ of the patients while they were being transported; therefore, it is important to send biological data in real time and to monitor arrhythmias or ST-T changes while transporting patients with AMI. 
Because we developed a system using mobile communications based on cellular phones, which are in widespread use, biological information, including 12-lead ECGs and inside-vehicle images from the ambulances can be sent continuously in real time, and this method is superior to others for the remote monitoring of arrhythmias and ST-T changes while transporting patients who are in the hyperacute period of AMI.

The door-to-balloon time was shorter for the patients in the MTS group. The most likely reason why MTS shortened the door-to-balloon time is that MTS helped cardiologists or emergency care physicians to recognize patients requiring prompt treatment with primary PCI. In this study, patients with STEMI who arrived at the hospital within 3 hours from the onset of AMI had a shorter door-to-balloon time in the MTS group than in the non-MTS group. This means that using MTS provides important information necessary for recognizing patients requiring primary PCI. In this study, patients who arrived at the hospital during after-hours had a shorter door-to-balloon time in the MTS group than in the non-MTS group. This observation suggests that biological information sent by MTS might provide the rationale needed to assemble staff for performing an emergency catheterization prior to the actual arrival of the patient at the hospital. In addition, MTS might help with the setup of the catheterization laboratory in crowed situations. In the present study, we did not observe a significant shortening of the door-to-balloon time among the working-hour cases because only 4 patients with STEMI arrived at the hospital within 3 hours from the onset of symptoms in the MTS group. However, if MTS was used for more patients in more crowed hospitals, MTS might shorten the setup time for the catheterization laboratory, resulting in a shortening of the door-to-balloon time.

\section{Study limitations}

The clinical use of MTS did not reduce the peak CK level or the in-hospital mortality rate in this study. The most possible reason was the relatively small number of patients in the MTS group. Another possible reason was the heterogeneity of the patients in both groups, since this study was not a randomized trial. Consequently, the EMS staff could choose not to use MTS so as to transfer patients with Killip class 4 as quickly as possible.

Some guidelines recommend minimizing the total ischemic time and to have a first medical contact-to-balloon time of within 90 minutes for patients with AMI. In the present study, we did not collect data from the EMS team; therefore, we could not analyze the first medical contact-to-balloon time. Consequently, evaluating the EMS activities with regard to the use of MTS will be examined as a next step in our research. Also, we enrolled all the patients who experienced AMI during this study period; therefore, some cases requested EMS assistance more than 6 hours after the onset of AMI symptoms. This point might ex- plain why the door-to-balloon time in the non-MTS group was more than 90 minutes. However, the first purpose of this study was to start applying MTS clinically and to investigate its effectiveness in a pre-hospital medical setting, so we enrolled all the patients with AMI during the study period in the analysis, as such cases were suitable for the use of MTS.

The average transportation time in this study was only 14 minutes; if MTS was used in an area where EMS requires a longer transportation time or fewer cardiovascular care units are available in the area, the door-to-balloon time and the total ischemic time are likely to be shortened through the use of MTS.

\section{Conclusions}

We developed and validated MTS, which utilizes mobile communications via cellular phones to transmit continuous biological information from an ambulance while the patient is being transported to a destination hospital in a pre-hospital situation. This study showed that MTS is an effective technology in a prehospital medical setting for the treatment of patients with AMI.

\section{Acknowledgement}

This research was supported by a research grant for Cardiovascular Disease (H19-Shinkin-03 Study for the establishment of a prehospital system for AMI and stroke) from the Ministry of Health, Labor and Welfare, Japan.

\section{References}

1) O'Connor RE, Bossaert L, Arntz HR, et al: Part 9: Acute coronary syndromes: 2010 International Consensus on Cardiopulmonary Resuscitation and Emergency Cardiovascular Care Science With Treatment Recommendations. Circulation 2010; 122(16 Suppl 2): S422-S465

2) Antman EM, Hand M, Armstrong PW, et al: 2007 Focused Update of the ACC/AHA 2004 Guidelines for the Management of Patients With ST-Elevation Myocardial Infarction: a report of the American College of Cardiology/American Heart Association Task Force on Practice Guidelines: developed in collaboration With the Canadian Cardiovascular Society endorsed by the American Academy of Family Physicians: 2007 Writing Group to Review New Evidence and Update the ACC/AHA 2004 Guidelines for the Management of Patients With ST-Elevation Myocardial Infarction, Writing on Behalf of the 2004 Writing Committee. Circulation 2008; 117: 296-329

3) Ting HH, Krumholz HM, Bradley EH, et al: Implementation and integration of prehospital ECGs into systems of care for acute coronary syndrome: a scientific statement from the American Heart Association Interdisciplinary Council on Quality of Care and Outcomes Research, Emergency Cardiovascular Care Committee, Council on Cardiovascular Nursing, and Council on Clinical Cardiology. Circulation 2008; 118: 1066-1079

4) Diercks DB, Kontos MC, Chen AY, et al: Utilization and impact 
of pre-hospital electrocardiograms for patients with acute STsegment elevation myocardial infarction: data from the NCDR (National Cardiovascular Data Registry) ACTION (Acute Coronary Treatment and Intervention Outcomes Network) Registry. J Am Coll Cardiol 2009; 53: 161-166

5) Garvey JL, MacLeod BA, Sopko G, et al: Pre-hospital 12-lead electrocardiography programs: a call for implementation by emergency medical services systems providing advanced life support-National Heart Attack Alert Program (NHAAP) Coordinating Committee; National Heart, Lung, and Blood Institute (NHLBI); National Institutes of Health. J Am Coll Cardiol 2006; 47: 485-491

6) Otsuka Y, Yokoyama H, Nonogi H: Novel mobile telemedicine system for real-time transmission of out-of-hospital ECG data for ST-elevation myocardial infarction. Catheter Cardiovasc Interv 2009; 74: 867-872

7) Kimura E, Norihiko T, Ishihara K. Development MFER (Medical waveform Format Encoding Rules) parser. AMIA Annu Symp Proc 2006: 985

8) Nissen SE, Brush JE, Krumholz HM: President's page: GAPD2B: an alliance for quality. J Am Coll Cardiol 2006; 48: 1911-1912

9) Cannon CP, Gibson CM, Lambrew CT, et al: Relationship of symptom-onset-to-balloon time and door-to-balloon time with mortality in patients undergoing angioplasty for acute myocar- dial infarction. JAMA 2000; 283: 2941-2947

10) McNamara RL, Wang Y, Herrin J, et al: Effect of door-to-balloon time on mortality in patients with ST-segment elevation myocardial infarction. J Am Coll Cardiol 2006; 47: 2180-2186

11) Krumholz HM, Anderson JL, Brooks NH, et al: ACC/AHA clinical performance measures for adults with ST-elevation and non-ST-elevation myocardial infarction: a report of the American College of Cardiology/American Heart Association Task Force on Performance Measures (Writing Committee to Develop Performance Measures on ST-Elevation and Non-ST-Elevation Myocardial Infarction). J Am Coll Cardiol 2006; 47: 236-265

12) Dhruva VN, Abdelhadi SI, Anis A, et al: ST-Segment Analysis Using Wireless Technology in Acute Myocardial Infarction (STAT-MI) trial. J Am Coll Cardiol 2007; 50: 509-513

13) Sanchez-Ross M, Oghlakian G, Maher J, et al: The STAT-MI (ST-Segment Analysis Using Wireless Technology in Acute Myocardial Infarction) trial improves outcomes. JACC Cardiovasc Interv 2011; 4: 222-227

14) Sejersten M, Sillesen M, Hansen PR, et al: Effect on treatment delay of prehospital teletransmission of 12-lead electrocardiogram to a cardiologist for immediate triage and direct referral of patients with ST-segment elevation acute myocardial infarction to primary percutaneous coronary intervention. Am J Cardiol 2008; 101: 941-946 